\title{
Per-organ assessment of subject-induced susceptibility distortion for MR-only male pelvis treatment planning
}

\author{
Carri Glide-Hurst $^{1 *+} \mathbb{D}$, Siamak Nejad-Davarani ${ }^{1 \dagger}$, Steffen Weiss ${ }^{2}$, Weili Zheng ${ }^{3}$, Indrin J. Chetty ${ }^{1}$ and Steffen Renisch ${ }^{2}$
}

\begin{abstract}
Background: Patient-specific distortions, particularly near tissue/air interfaces, require assessment for magnetic resonance (MR) only radiation treatment planning (RTP). However, patients are dynamic due to changes in physiological status during imaging sessions. This work investigated changes in subject-induced susceptibility distortions to pelvic organs at different bladder states to support pelvis MR-only RTP.

Methods: Pelvises of 9 healthy male volunteers were imaged at 1.0 Tesla (T), 1.5 T, and 3.0 T. Subject-induced susceptibility distortion field maps were generated using a dual-echo gradient-recalled echo (GRE) sequence with $B_{0}$ field maps obtained from the phase difference between the two echoes acquired at several bladder volume states (3-4/subject, 32 overall). T2 turbo spin echo images were also acquired at each bladder state for organ delineation. Magnet central frequency was tracked over time. Distortion map differences and boxplots were computed to characterize changes within the clinical target volume (CTV), bladder, seminal vesicles, and prostate volumes.

Results: The time between the initial and final B0 maps was $42.6 \pm 13.9$ (range: 13.2-62.1) minutes with minimal change in magnet central frequency $(0.02 \pm 0.05 \mathrm{~mm}$ (range: $-0.06-0.12 \mathrm{~mm}$ )). Subject-induced susceptibility distortion across all bladder states, field strengths, and subjects was relatively small (1.4-1.9\% of all voxels in the prostate and seminal vesicles were distorted $>0.5 \mathrm{~mm}$ ). In the bladder, no voxels exhibited distortions $>1 \mathrm{~mm}$. An extreme case acquired at 3.0 T with a large volume of rectal air yielded $27.4-34.6 \%$ of voxels within the CTVs had susceptibility-induced distortions $>0.5 \mathrm{~mm}$ across all time points.
\end{abstract}

Conclusions: Our work suggests that subject-induced susceptibility distortions caused by bladder/rectal conditions are generally small and subject-dependent. Local changes may be non-negligible within the CTV, thus proper management of filling status is warranted. Future work evaluating the impact of multiple models to accommodate for extreme status changes may be advantageous.

\section{Background}

Images of high geometric fidelity enable accurate delineation of disease extent and proximity to organs at risk (OARs), which are essential for high-precision radiation treatment planning (RTP). However, the current standard of care for RTP is based on CT simulation (CT-SIM), which does not provide adequate soft tissue discrimination. This limitation has been addressed by registering diagnostic magnetic resonance imaging data (MRI) to

\footnotetext{
* Correspondence: churst2@hfhs.org

${ }^{+}$Carri Glide-Hurst and Siamak Nejad-Davarani contributed equally to this work. ${ }^{1}$ Department of Radiation Oncology, Henry Ford Cancer Institute, Detroit, MI 48202, USA

Full list of author information is available at the end of the article
}

CT-SIM datasets. This existing CT-SIM-based workflow relies on target and OAR definition on MRI and a transfer of contours to CT via image registration. MRI-CT co-registration introduces geometrical uncertainties of $2 \mathrm{~mm}$ for prostate patients [1,2]. Importantly, these errors are systematic, persist throughout treatment, shift high dose regions away from the target [3] and could lead to a geometric miss that compromises tumor control. Thus, there has been a strong interest to move toward an MR-only RTP workflow to eliminate redundant CT scans (reducing radiation dose, patient time, and imaging costs), streamline clinical efficiency, and importantly, MR-only RTP would entirely circumvent systematic co-registration uncertainties [4-6]. 
MRI has been limited by known geometric distortions arising from two major components: system-level (arising from gradient nonlinearity (GNL) in the spatial encoding gradients $[7,8]$ and $\mathrm{B}_{0}$ field inhomogeneities) and patient-level (chemical shift artifacts and susceptibility) [9]. Currently, GNL distortion corrections are built into the MRI reconstruction software. While our preliminary results at $1.0 \mathrm{~T}$ showed that residual GNL-after vendor corrections-was non-negligible and required additional corrections before MR-only RTP can be implemented [10], closed bore magnets have shown clinically acceptable GNL within the useable field of view (FOV) $[11,12]$. Patient-level distortions, on the other hand, are object- and sequence-dependent and increase with field strength, requiring patient-specific corrections. Susceptibility differences are most apparent near tissue/ air interfaces due to local variations in the induced magnetic field and have been reported to be up to $4 \mathrm{~mm}$ at the sinus/tissue interface in the brain at 3.0 $\mathrm{T}$ [9].

Few studies have investigated the impact of subject-induced susceptibility distortion in the pelvis. Recently, Tyagi et al. measured distortions using $B_{0}$ maps (i.e., the difference of two phase images measured at different echo times (TEs)) and found that the distortions within the prostate were $<0.5 \mathrm{~mm}$ for a 20 patient cohort, however other organs and multiple time points were not studied [13]. Stanescu et al. and Lundman et al. performed simulation studies to investigate susceptibility distortions in the pelvis and found the magnitude was lower than other sites such as lung or head and neck $[14,15]$. In another study, Kemppainen et al. investigated patient specific geometric distortions in the PTV, bladder and rectum of four pelvic cancer subjects on a $1.5 \mathrm{~T}$ scanner and reported a distortion of less than $1 \mathrm{~mm}$ in these organs [16]. However, in addition to susceptibility induced distortions, pelvic patients may experience changing transient anatomy (e.g., bladder and rectal filling) that can occur during long MR image acquisition times. These status changes may result in geometric inconsistencies as well as local subject-induced susceptibility distortion changes (termed "susceptibility distortions" in this work) at air/tissue interfaces. This work sought to characterize changes in susceptibility distortions in pelvic organs arising from transient anatomy during MRI examinations to support an MR-only RTP workflow in the pelvis.

\section{Methods and materials}

\section{Subject population}

Nine healthy male volunteers (age: $43 \pm 10.1$ years, range 25-61 years; weight: $78.8 \pm 9.6 \mathrm{~kg}$ ) were consented to research studies managed by local Institution Review Boards. Three volunteers were scanned using a large, rigid 8-element phased array coil on a $1.0 \mathrm{~T}$ Panorama High
Field Open Magnetic Resonance System (1.0 T Panorama HFO; Philips Healthcare, Best, The Netherlands) equipped with flat table top (Civco, Orange City, IA) and external laser system. Three healthy male volunteers were scanned at $1.5 \mathrm{~T}$ (Philips Achieva, 32-element torso coil) and the last three on a $3.0 \mathrm{~T}$ (Philips Ingenia, 32-element coil in the patient bed and anterior array) with standard concave table tops and using the magnet bore lasers for positioning. Figure 1 illustrates the experimental design for image acquisition for all cases. In brief, subjects were instructed to void their bladder and then consumed $\sim 600 \mathrm{ml}$ of water. They were positioned supine and sequences were acquired at several states: (State 1) empty bladders, (States $2-3$ ) partially full bladders, and (States 3-4) full bladders at each field strength. The total imaging time on all scanners was $\sim 45 \mathrm{~min}$. Considering that lower field strengths have longer acquisition times, only three T2-weighted and B0 image sets were acquired at $1.0 \mathrm{~T}$. However, for $1.5 \mathrm{~T}$ and $3.0 \mathrm{~T}$, shorter sequence times allowed for the acquisition of additional bladder states within this time. Therefore, 3-4 evaluable states were used per subject based on field strength with a total of 32 evaluable time points for the cohort. After two full sets of acquisitions were acquired, an additional $300-600 \mathrm{ml}$ of water was consumed while the subject was on the table with no repositioning as illustrated by Fig. 1. In this manner, a range of bladder filling states (i.e. between interim and extreme states of bladder filling) could be investigated. A similar workflow can also be used to create a library of radiation treatment plans for actual patients, similar to a study that was previously done for cervical cancer patients [17].

\section{Subject-induced susceptibility distortion maps}

For each time point, T2-weighted (T2) turbo spin echo images were also acquired as these are most commonly used for delineation of the prostate gland $[18,19]$ and cervical cancer [20]. The bandwidth of the T2 sequence was chosen to optimize signal-to-noise ratio (SNR) as is common for this task. Field mapping was performed using a dual-acquisition gradient echo (GRE) sequence. FOVs were chosen to cover the entire body laterally and the pelvic region of interest in feet-head direction. Shim settings were held constant for each subject across the entire acquisition time with sequence parameters shown in Table 1. At each field strength, GRE TEs were set to yield two in-phase images, higher bandwidths were used to minimize the chemical shift related distortion, and voxel sizes were selected based on tradeoffs between resolution and acquisition time, which were also consistent with the literature [9]. Maps of the phase difference $(\Delta \phi)$ were reconstructed by evaluating the phase after complex division of the complex data from two echoes. The phase of the complex ratio equals to the phase difference between the two images. Phase difference maps 


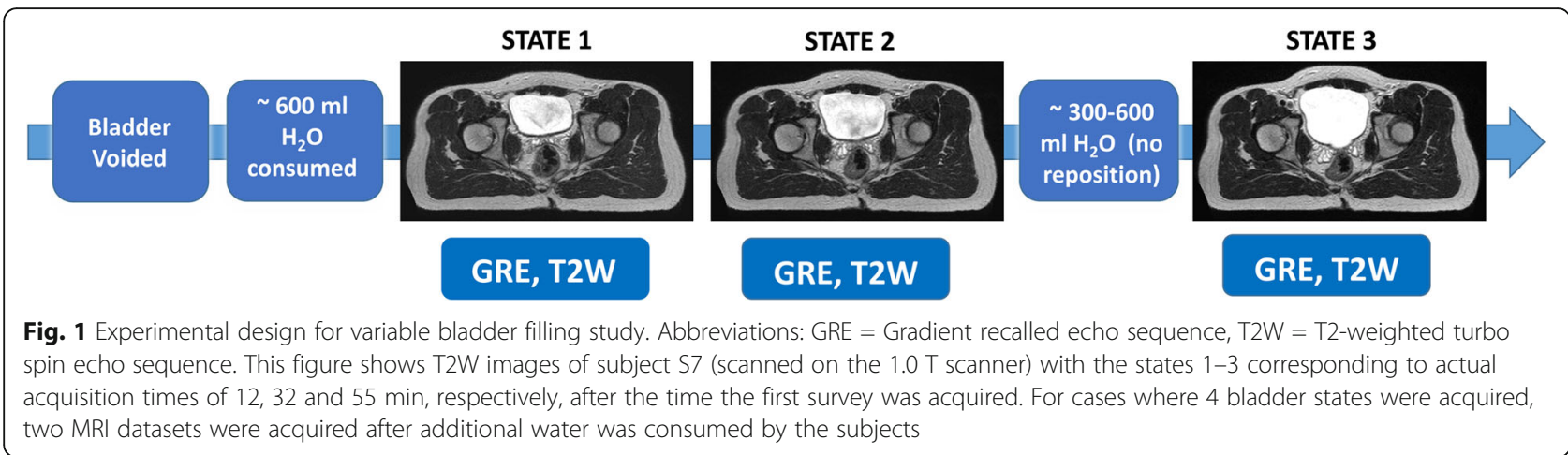

were unwrapped using Prelude in the Functional Magnetic Resonance Imaging of the Brain (FMRIB) Software Library (FSL, Analysis Group, FMRIB, Oxford, UK). $B_{0}$ field maps were obtained from the phase difference $\Delta \phi$ evolved between the two echoes with time difference $\square £$ TE: $\Delta B_{0}=\frac{\Delta \phi}{2 \pi \gamma \Delta T E}$ ( $\gamma$ is the gyromagnetic ratio of hydrogen) [21]. This study focuses on a Cartesian non-echo-planar T2 sequence for delineation. For such a sequence, susceptibility-based local changes of the resonance frequency directly transfer into local displacements along the frequency encoding or read-out direction, whereas the phase encoding direction remains unaffected. To convert the $B_{0}$ field map to a distortion map ( $\mathrm{x}$-displacement) based on the T2 sequence, the following equation was used:

$$
\Delta \mathrm{x}=\frac{\Delta \mathrm{B}_{0}}{\mathrm{G}_{x}}=\frac{\Delta f_{0}}{B W_{f}} \Delta \mathrm{V}_{\mathrm{x}}=\frac{\Delta \phi}{2 \pi \Delta T E * B W_{f}} \Delta \mathrm{V}_{\mathrm{x}}
$$

with following T2 sequence parameters: $G_{x}$ is the readout gradient, $\Delta \mathrm{x}$ is $\mathrm{x}$-displacement along the frequencyencoding direction; $B W_{\mathrm{f}}$ is the acquisition pixel bandwidth $(\mathrm{Hertz}(\mathrm{Hz}) /$ pixel) in the frequency encoding direction and $\Delta \mathrm{V}_{\mathrm{x}}$ is pixel size in the frequency encoding direction. Other sequences will require sequence-specific corrections that are beyond the scope of this work and detailed in the literature [21].

It is important to note that the subjects were not repositioned between acquisitions. To further minimize effects of any displacement of the subjects between acquisitions, magnitude images from the first echo of all GRE and T2 datasets were registered to the first image set using FMRIB's Linear Image Registration Tool module in FSL [22, 23]. Six parameter (translation and rotation) rigid registration was performed using nearest neighbor interpolation and mutual information as the cost function. Visual inspection of bony alignment was performed for each case. The resultant transformations were then applied to the corresponding $\mathrm{B}_{0}$ field maps to perform voxel-by-voxel comparisons.

To quantify susceptibility effects in organs of interest, the prostate, seminal vesicles, and bladder were delineated on the corresponding T2-weighted dataset at of each bladder state by a single physician. The clinical target volume (CTV) was defined as the union of the prostate and proximal $1 \mathrm{~cm}$ of the seminal vesicles as consistent with our clinical practice [24]. The delineated organs were used as a mask on the corresponding distortion map to quantify susceptibility-induced distortions for each organ across images from all bladder states via box plots and corresponding descriptive statistics. One-way analysis of variance (ANOVA) (with posthoc

Table $1 \mathrm{MRI}$ sequence parameters used in this study. The dual-acquisition gradient echo (GRE) sequence was used for field mapping and the T2 images were acquired for delineation of the organs. $x, y$ and $z$ indicate the axes in the left-right, anterior-posterior and superior-inferior directions respectively

\begin{tabular}{llllllllll}
\hline Field Strength (T) & Sequence & $\begin{array}{l}\text { Acquisition } \\
\Delta \mathrm{V}_{x} / \Delta \mathrm{V}_{\mathrm{y}} / \Delta \mathrm{V}_{z} \\
(\mathrm{~mm})\end{array}$ & Bandwidth (Hz/pixel) & a & TR (ms) & TE1(ms) & TE2(ms) & FOV (mm) & Turbo Factor \\
\hline 1.0 & GRE & $1.41 / 1.41 / 3$ & 975 & $10^{\circ}$ & 15.87 & 6.91 & 13.81 & $474.19 \times 474.19 \times 300$ & - \\
& T2 & $1.49 / 1.6 / 2.5$ & 202 & $90^{\circ}$ & 5070 & 80 & - & $397.89 \times 397.89 \times 250$ & 24 \\
1.5 & GRE & $2.9 / 2.9 / 4$ & 543 & $10^{\circ}$ & 11.19 & 4.61 & 9.22 & $416.90 \times 416.90 \times 200$ & - \\
& T2 & $0.96 / 0.96 / 2.5$ & 207 & 90 & 4620 & 100 & - & $180 \times 180 \times 84$ & 17 \\
3.0 & GRE & $2.9 / 2.9 / 4$ & 1078 & $10^{\circ}$ & 2.30 & 2.30 & 4.61 & $416.90 \times 416.90 \times 200$ & - \\
& T2 & $0.96 / 0.96 / 2.5$ & 218 & $90^{\circ}$ & 4295 & 100 & - & $180 \times 180 \times 84$ & 17 \\
\hline
\end{tabular}


Bonferroni adjustments to address multiple comparisons) were performed to evaluate statistically significant differences in susceptibility-induced distortion between physiological states (i.e., empty vs. partially full bladder, empty vs. full bladder, etc.) within each subject.

To employ the distortion measurement methodology proposed here, the resonant frequency ( $\mathrm{fO}$ ) of the scanner is assumed to be constant over the entire imaging acquisition session. However, scanner resonant frequencies may change due to temperature changes, warming of electronics, or variations in the subject composition (i.e. presence of air, tissue, etc.) in or near the volume used for f0 determination [25]. To characterize these variations, the $\mathrm{f} 0$ was tabulated from the image header of the B0 map sequence for each subject and field strength. The f0 is the optimal RF excitation frequency that is automatically measured at the beginning of each MRI scan based on a subvolume centered within the imaging FOV. These data are logged by the scanner in the DICOM header and were used in this work to assess the stability of the f0-determination. For the shim procedure, the shimming is held constant for each volunteer. The frequency (in $\mathrm{Hz}$ ) was converted to units of T2 distortion using Eq. 1.

\section{Results}

\section{Central frequency drift}

On average, the time between the initial and final B0 maps (time point 3 or 4 depending on the subject) was $42.6 \pm$ 13.9 (range: 13.2-62.1) minutes. As shown in Fig. 2, the central frequency drift effect over the imaging sessions across all magnets was generally negligible $(0.02 \pm$ $0.05 \mathrm{~mm}$ (range: $-0.06-0.12 \mathrm{~mm}$ )). The maximum f0 shift $(16 \mathrm{~Hz})$ occurred for the $1.0 \mathrm{~T}$ scanner over 50 min, which was converted to a T2 distortion of $0.12 \mathrm{~mm}$ for time points 2 and 3 (Subject 8 in Fig. 2).
Despite the larger central frequency drift for this case, the corresponding distortion in the PTV was shown to increase at the 2nd time point and subsequently decrease at the 3rd time point.

\section{Magnitude and distribution of patient-specific distortions}

Figure 3 summarizes the overall distortions within the prostate and seminal vesicles. Subjects 7, 8, and 9 acquired at $1.0 \mathrm{~T}$ demonstrated the smallest susceptibility distortions. Notably, Subject 2, acquired at $3.0 \mathrm{~T}$, not only showed the largest susceptibility but also the largest variation of the magnitude of the bladder volume. There was a significant effect of bladder state on organ-specific distortion at the $p<0.05$ level for 37 out of 42 combinations of matched states for the prostate, seminal vesicles, and bladder. All pairwise comparisons between the initial and final states were statistically different for all organs studied. Even so, the overall magnitude of distortion yielded only 1.4$1.9 \%$ of all voxels in the prostate and seminal vesicles distorting $>0.5 \mathrm{~mm}$ across the cohort. When Subject 2 was excluded, the overall distortion was negligible for all other cases $(<0.2 \%$ and $<0.03 \%$ of all voxels distorted $>0.5 \mathrm{~mm}$ within the seminal vesicle and prostate, respectively). In the bladder, no voxels exhibited distortions $>1 \mathrm{~mm}$. Again, the largest distortion in the bladder occurred for Subject 2 with $1.3-2.2 \%$ of voxels distorting $>0.5 \mathrm{~mm}$ across all bladder states studied. For this case shown in Fig. 5, large pockets of rectal air were observed at the first state that did not fully resolve over the entire acquisition.

Overall, distortion magnitude was small although the voxel-based distributions illustrate that the overall ranges and distortion changes based on bladder state were subject-specific. Figure 4 summarizes the CTV distributions for all time points in the cohort. Figure 5

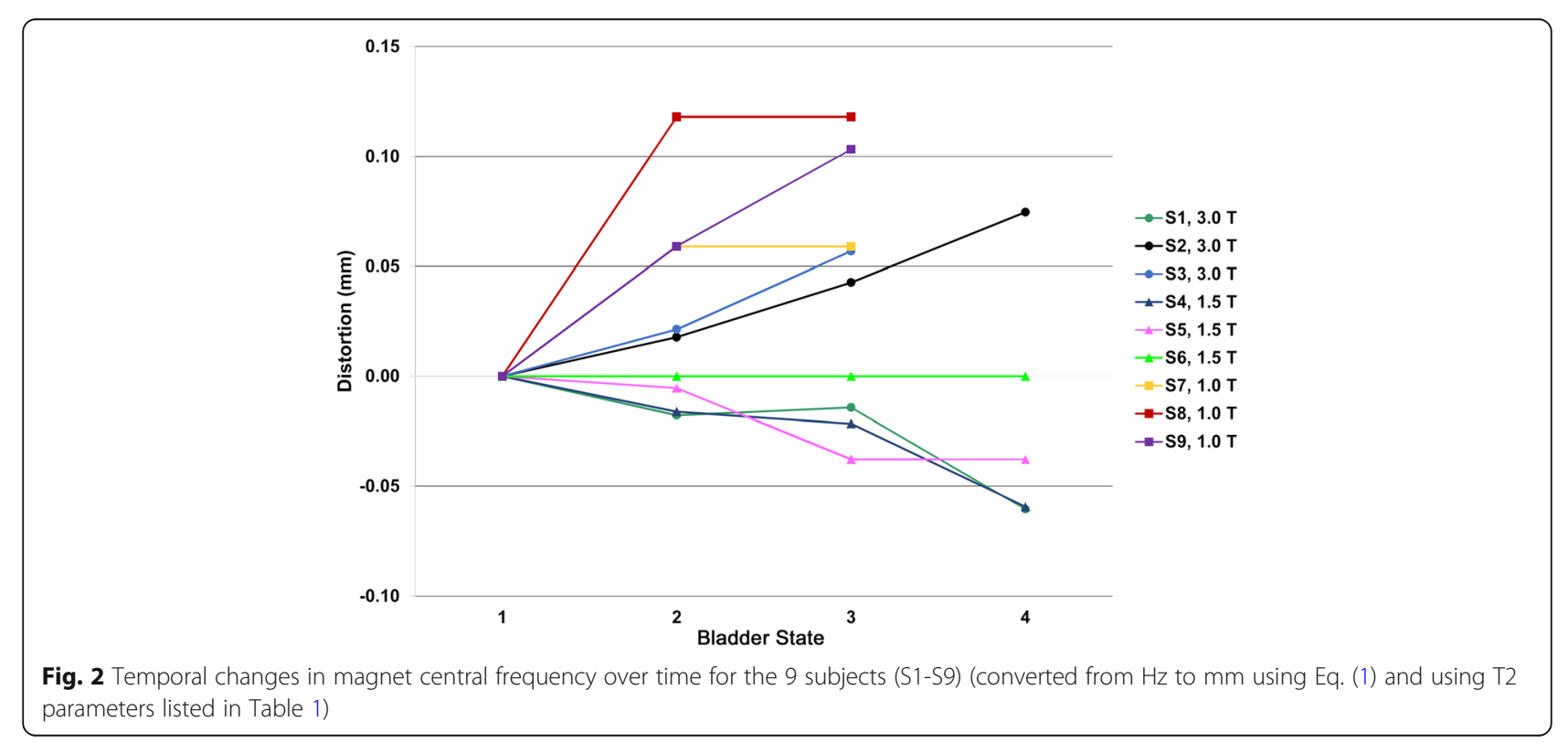



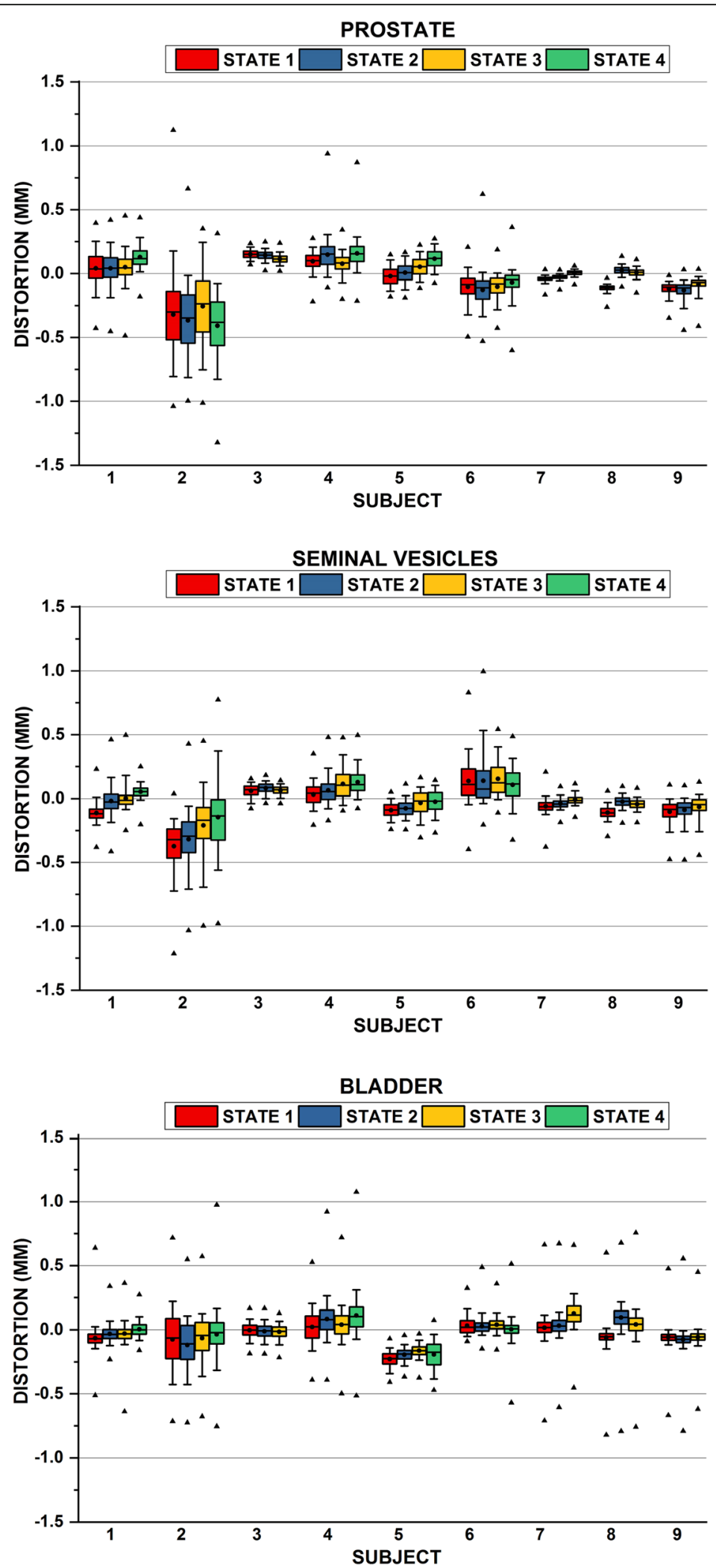

Fig. 3 (See legend on next page.) 
(See figure on previous page.)

Fig. 3 Distribution of patient-induced susceptibility effects in the prostate (top), seminal vesicles (middle), and bladder (bottom) for bladder state (S1 Empty bladder, S3 or S4 Full bladder). Boxplots, line, and dot indicate the interquartile range (25-75\%), median, and mean respectively. Whiskers indicate 5th and 95th percentile, $\boldsymbol{\Delta}$ indicate the minimum and maximum values. Subjects 1-3 were acquired at 3.0 T, 4-6 were acquired at 1.5 T, and 7-9 were acquired at 1.0 T

illustrates local distortion differences arising from rectal/ bowel changes induced by bladder filling for a subject at each field strength to highlight different characteristics in the subject population. For Subject 2, changes in bowel air were observed, with large amounts of rectal air influencing distortions within the CTV ( 27.4 to 35.7\% of the voxels yielded patient-specific susceptibility distortions $>0.5 \mathrm{~mm}$, largely at the rectum/bowel gas and tissue interfaces). Subject 6 was selected at $1.5 \mathrm{~T}$ as it showed the largest distortions at this field strength (range $=0.8$ to $1.1 \mathrm{~mm}$ within the CTV). Finally, Subject 9 data acquired at $1.0 \mathrm{~T}$ illustrated that a very large increase in bladder volume did not influence the rectal status for this case, and thus susceptibility-induced distortions within the CTV remained stable whereas local distortions in the bowel were more apparent.

\section{Discussion}

As we move toward MRI-only treatment planning, it becomes important to characterize distortion magnitude that may arise in this new workflow. This work sought to quantify patient-specific distortion in the pelvis and considered geometric changes that may occur in the delineated organs based on susceptibility induced distortions due to bladder and rectal status changes occurring over long MRI acquisition times. The results of this work may also extend to MR-guided radiation therapy, particularly at higher field strengths where subject-induced distortions are expected to be higher. While MRI acquisition times were long in this study, the baseline variation of the fo determination during that time was negligible $(0.02 \pm$ $0.05 \mathrm{~mm}, \sim 0.12 \mathrm{~mm}$ in the most extreme case). In our study, the resonance frequency (f0) was held constant across the acquisition session. In the pelvis, compositional changes in bowel and rectal gas may occur over an image acquisition session and thus impact the volume used for fo determination, thus it is recommended that this should be held constant for magnetic resonance simulation examinations. Recently, Wang et al. performed repeat acquisition of field maps for 17 brain subjects and found a within-subject standard deviation of $\sim 0.2 \mathrm{~mm}$ displacement in the frequency-encoding direction of 3D T1-weighted images [9]. The authors concluded that this variation was small and possibly due to eddy current decay in their 3.0 T magnet, which can also be a contributing effect to variations in susceptibility over time. In our study of the male pelvis, physiological status changes across 3 field strengths caused more marked impact on local susceptibility than what was observed for the repeat measurements in the brain.

While statistically significant differences were found in 37 out of 42 comparisons for the prostate and seminal vesicles, overall distortion magnitude was quite low $(<2 \%$ of all voxels distorted $>0.5 \mathrm{~mm}$ ). Subject 2 exhibited the largest distortions in the prostate and seminal vesicles. When Subject 2 was omitted from the cohort, $<0.2 \%$ of all prostate and seminal vesicles voxels across all states had subject-induced susceptibilities $>0.5 \mathrm{~mm}$. This work considered subject-induced distortions in several male pelvic organs, however the femoral heads were not analyzed. Recent work published by Adjeiwaah et al. simulated expected subject-induced distortions with similar bandwidths at $244 \mathrm{~Hz} /$ pixel and found that median distortions were $\sim 0.5 \mathrm{~mm}$ with $\sim 75 \%$ of all distortions in the femoral heads were $<1 \mathrm{~mm}$ [26]. Also, Tyagi et al. evaluated patient-induced susceptibility distortion in the pelvis in a cohort of 20 prostate patients acquired at 3.0 T. They found the mean distortion within the prostate for a single acquisition dataset to be $0.2 \mathrm{~mm}$ (range: $-0.62-0.35 \mathrm{~mm}$ ) [13]. These results were of similar magnitude to our population results although their calculations were based on full bladder conditions controlled by a Foley catheter. In their study, the mDixon sequence with a relatively high acquisition pixel bandwidth used to generate synthetic CTs for treatment planning using commercially available software was evaluated whereas our study was focused on the T2-weighted sequences used for delineation. Bandwidths used in this study were selected to optimize SNR and are consistent with literature on T2-weighted pelvis acquisitions [13, 26]. Eq. 1 highlights that distortion due to susceptibility effects is dependent on the frequency-encoding bandwidth and field strength. This effect can be reduced by increasing the acquisition bandwidth at the expense of reducing SNR. Our results can be extrapolated to other T2-weighted sequences with different acquisition parameters based on Eq. 1.

Susceptibility distortions scale with field strength and size of the inhomogeneity [14]. This was observed in our study, where the magnitudes of local distortions in the CTV were largest for subjects at higher field strengths and with larger rectal gas volumes. Thus, it can be expected that when the volume of rectal air increases, susceptibility-related distortions in the abutting CTV would also increase, which was consistent with our results. It was found that subjects with no rectal air near had minimal susceptibility distortion changes over different bladder volume states. Similarly, 


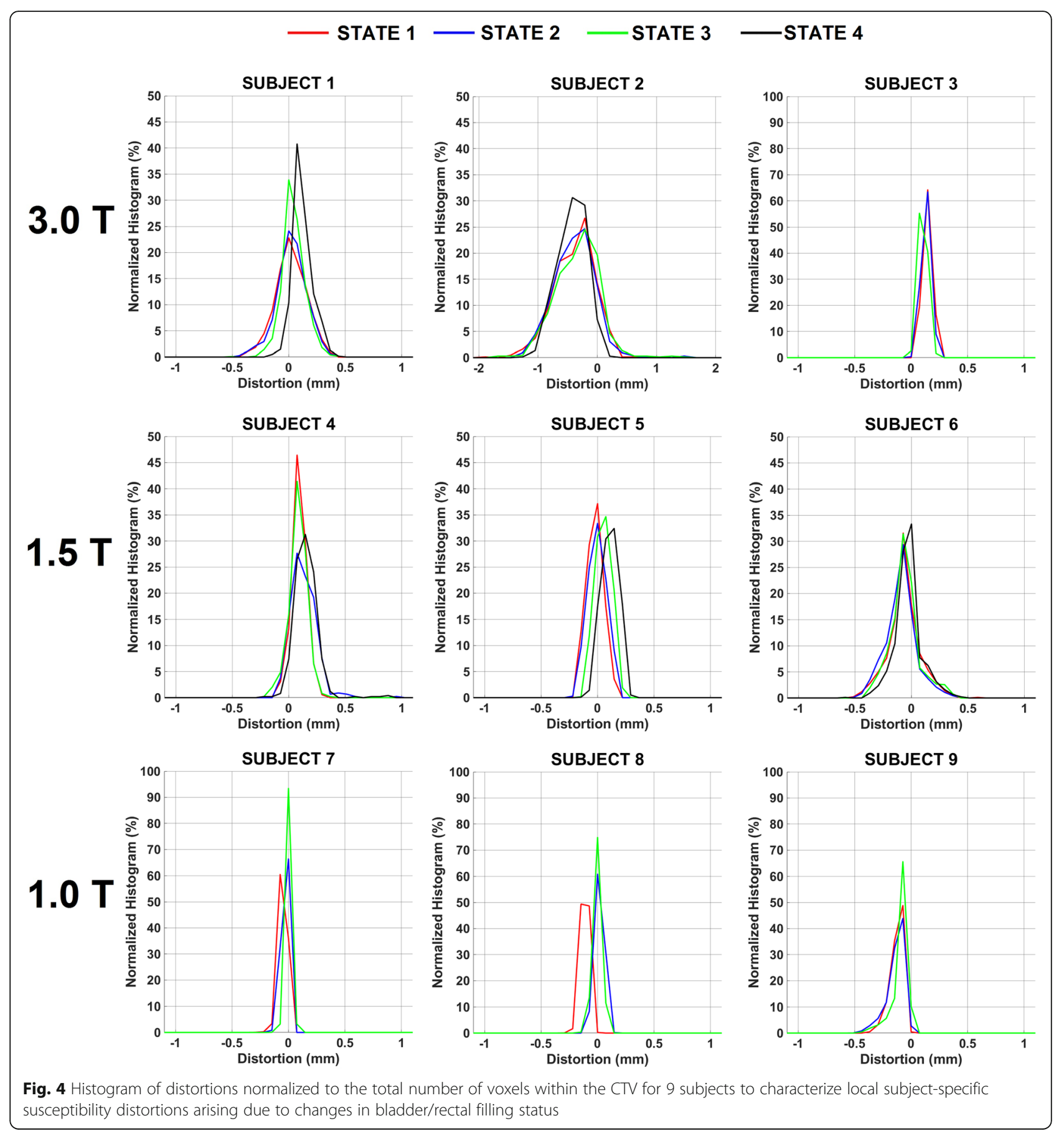

subjects acquired at the lowest field strength of $1.0 \mathrm{~T}$ had no appreciable differences in susceptibility effects using the different models. However, in a worst-case scenario (Subject 2 acquired at $3.0 \mathrm{~T}$ with large rectal status changes between time points), distortion differences within the CTV were appreciable. This suggests that had the first set of susceptibility maps been used to correct for susceptibility distortions, corrections would have been applied to anatomy that was no longer at the same state and location.
One limitation of this study was that data was obtained on healthy volunteers. However, intermediate and extreme physiological states, ranging from empty to full bladders, were considered. Fig. 5 illustrated that the presence of large pockets of rectal air introduced the largest susceptibility distortions although almost all voxels distorted $<1 \mathrm{~mm}$. Recently, Adjeiwaah et al. simulated dosimetric differences due to patient-induced susceptibility at various bandwidths for 17 prostate cases 


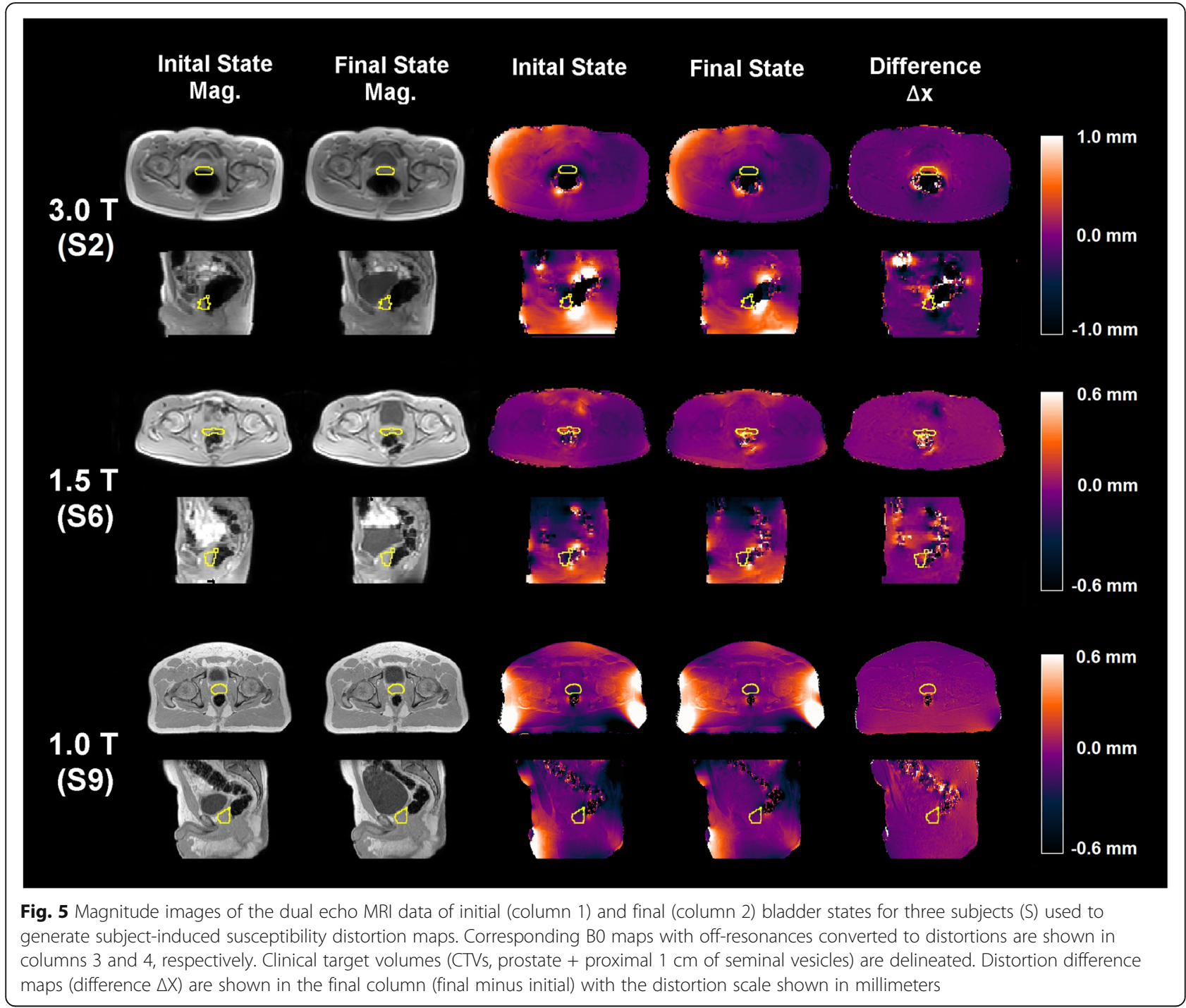

and found clinically acceptable plan quality with relative dose differences of $<0.5 \%$ in the PTV [26], suggesting that distortions may not yield dosimetric differences.

This work was not designed to address GNL distortions, although applying vendor distortion corrections and centering the area of interest near magnet isocenter can reduce GNL to $<1 \mathrm{~mm}$ for most magnets [11, 27]. While distortion correction maps can be applied, our work has illustrated the potential distortion changes arising from different organ states, thereby complicating the use of a single distortion correction map in the pelvis. This suggests that if corrections are to be made, an important quality assurance step will include evaluating spatial alignment of air-filled organs. Because patient-induced susceptibility distortions will arise near tissue/air interfaces such as the prostate/rectal interface where high dose gradients typically exist, it may be advantageous to correct for the distortions via an inverse warping and Jacobian scaling technique as described in the literature [10, 28]. This interface is critical in RTP because margins are typically reduced to decrease the risk of rectal toxicity [29] and volumetric image-guided radiation therapy alignment is emphasized in this region [24]. Another approach would be to ensure proper margins are applied to accommodate this uncertainty. Overall, organ-specific subject-induced distortions quantified in this work were small but may be clinically significant based on the location.

\section{Conclusions}

Our work suggests that subject-specific distortion differences caused by transient gas are generally small and subject-dependent. However, local changes may be non-negligible near interfaces, thus proper management of filling status is warranted. 


\section{Abbreviations}

ANOVA: Analysis of variance; $B_{0}$ map: Map of the $B_{0}$ field created using the difference of two phase images measured at different echo times; CT-SIM: Computed tomography simulation; CTV: Clinical target volume; f0: Resonant frequency; FMRIB: Functional magnetic resonance imaging of the brain; FOV: Field of view; FSL: FMRIB Software library; GNL: Gradient nonlinearity; GRE: Gradient echo; Hz: Hertz; kg: Kilograms; mm: Millimeter; MR: Magnetic resonance; OAR: Organ at risk; PTV: Planning target volume; RTP: Radiation treatment planning; SNR: Signal-to-noise ratio; T: Tesla; T2: T2-weighted; TE: Echo time

\section{Funding}

Data collection, analysis, and writing of the manuscript were all supported by the National Cancer Institute of the National Institutes of Health under Award Number R01CA204189.

\section{Availability of data and materials}

The datasets during and/or analysed during the current study available from the corresponding author on reasonable request.

\section{Authors' contributions}

SW, SR, CGH, SND, and WZ collected and analyzed the subject MRI data. IJC assisted with data interpretation and analysis. All authors read and approved the final manuscript.

\section{Ethics approval and consent to participate}

Written informed consent was obtained for all research subjects in this study at both institution's Institutional Review/Ethics Boards.

\section{Consent for publication}

Not applicable.

\section{Competing interests}

CGH, SR: Research partially supported by the National Cancer Institute of the National Institutes of Health under Award Number R01CA204189.

SW and SR are employees at Philips Healthcare, Research in Hamburg, Germany.

CGH reports research funding with Philips Healthcare and ViewRay, Inc. IJC reports research agreements with Philips Healthcare and Varian Medical Systems.

SND and WZ declare that they have no competing interests.

\section{Publisher's Note}

Springer Nature remains neutral with regard to jurisdictional claims in published maps and institutional affiliations.

\section{Author details}

'Department of Radiation Oncology, Henry Ford Cancer Institute, Detroit, MI 48202, USA. ${ }^{2}$ Department of Digital Imaging, Philips Research Laboratories, 22335 Hamburg, Germany. ${ }^{3}$ Department of Radiation Oncology, William Beaumont Hospital, Royal Oak, MI 48073, USA.

\section{Received: 16 April 2018 Accepted: 27 July 2018}

\section{Published online: 15 August 2018}

\section{References}

1. Dean CJ, Sykes JR, Cooper RA, et al. An evaluation of four CT-MRI coregistration techniques for radiotherapy treatment planning of prone rectal cancer patients. Br J Radiol. 2012;85:61-8.

2. Korsager AS, Carl J Riis Ostergaard L. Comparison of manual and automatic MR-CT registration for radiotherapy of prostate cancer. J Appl Clin Med Phys. 2016;17:294-303.

3. Van Herk M. Errors and margins in radiotherapy. Semin Radiat Oncol. 2004;14:52-64.

4. Tyagi N, Fontenla S, Zelefsky M, et al. Clinical workflow for MR-only simulation and planning in prostate. Radiat Oncol. 2017;12:119.

5. Owrangi AM, Greer PB, Glide-Hurst CK. MRI-only treatment planning: benefits and challenges. Phys Med Biol. 2018;63:05TR01.

6. Edmund JM, Nyholm T. A review of substitute $C T$ generation for MRI-only radiation therapy. Radiat Oncol. 2017;12:28.
7. Baldwin LN, Wachowicz K, Thomas SD, et al. Characterization, prediction, and correction of geometric distortion in 3 T MR images. Med Phys. 2007; 34:388.

8. Wang D, Doddrell DM, Cowin G. A novel phantom and method for comprehensive 3-dimensional measurement and correction of geometric distortion in magnetic resonance imaging. Magn Reson Imaging. 2004;22: $529-42$.

9. Wang H, Balter J, Cao Y. Patient-induced susceptibility effect on geometric distortion of clinical brain MRI for radiation treatment planning on a 3T scanner. Phys Med Biol. 2013;58:465.

10. Price RG, Kadbi M, Kim J, et al. Technical note: characterization and correction of gradient nonlinearity induced distortion on a 1.0 T open bore MR-Sim. Med Phys. 2015;42:5955-60.

11. Torfeh T, Hammoud R, Perkins G, et al. Characterization of 3D geometric distortion of magnetic resonance imaging scanners commissioned for radiation therapy planning. Magn Reson Imaging. 2016;34:645-53.

12. Huang KC, Cao Y, Baharom U, et al. Phantom-based characterization of distortion on a magnetic resonance imaging simulator for radiation oncology. Phys Med Biol. 2016;61:774.

13. Tyagi N, Fontenla S, Zhang J, et al. Dosimetric and workflow evaluation of first commercial synthetic CT software for clinical use in pelvis. Phys Med Biol. 2017:62:2961-75.

14. Stanescu T, Wachowicz K, Jaffray DA. Characterization of tissue magnetic susceptibility-induced distortions for MRIgRT. Med Phys. 2012:39:7185-93.

15. Lundman JA, Bylund M, Garpebring A, et al. Patient-induced susceptibility effects simulation in magnetic resonance imaging. Phys Imaging Radiat Oncol. 2017;1:41-5.

16. Kemppainen $\mathrm{R}$, Suilamo $\mathrm{S}$, Tuokkola $\mathrm{T}$, et al. Magnetic resonance-only simulation and dose calculation in external beam radiation therapy: a feasibility study for pelvic cancers. Acta Oncol. 2017;56:792-8.

17. Sharfo AW, Breedveld S, Voet PW, et al. Validation of fully automated VMAT plan generation for library-based plan-of-the-day cervical Cancer radiotherapy. PLoS One. 2016:11:e0169202.

18. Paulson ES, Erickson B, Schultz C, et al. Comprehensive MRI simulation methodology using a dedicated MRI scanner in radiation oncology for external beam radiation treatment planning. Med Phys. 2015;42:28.

19. Sannazzari G, Ragona R, Ruo Redda M, et al. CT-MRI image fusion for delineation of volumes in three-dimensional conformal radiation therapy in the treatment of localized prostate Cancer. Br J Radiol. 2002;75:603-7.

20. Haie-Meder C, Pötter R, Van Limbergen E, et al. Recommendations from Gynaecological (GYN) GEC-ESTRO working group (I): concepts and terms in 3D image based 3D treatment planning in cervix cancer brachytherapy with emphasis on MRI assessment of GTV and CTV. Radiother Oncol. 2005 74:235-45.

21. Baldwin LN, Wachowicz K, Fallone BG. A two-step scheme for distortion rectification of magnetic resonance images. Med Phys. 2009:36:3917-26.

22. Jenkinson M, Smith S. A global optimisation method for robust affine registration of brain images. Med Image Anal. 2001:5:143-56.

23. Jenkinson M, Bannister P, Brady M, et al. Improved optimization for the robust and accurate linear registration and motion correction of brain images. Neuroimage. 2002;17:825-41.

24. Wen N, Glide-Hurst C, Nurushev T, et al. Evaluation of the deformation and corresponding dosimetric implications in prostate cancer treatment. Phys Med Biol. 2012:57:5361.

25. Benner T, van der Kouwe AJ, Kirsch JE, et al. Real-time RF pulse adjustment for BO drift correction. Magn Reson Med. 2006;56:204-9.

26. Adjeiwaah $M$, Bylund $M$, Lundman JA, et al. Quantifying the effect of $3 T$ magnetic resonance imaging residual system distortions and patientinduced susceptibility distortions on radiation therapy treatment planning for prostate Cancer. Int J Radiat Oncol Biol Phys. 2018;100:317-24.

27. Price $R G$, Knight RA, Hwang $K$, et al. Optimization of a novel large field of view distortion phantom for Mr-only treatment planning. J Appl Clin Med Phys. 2017;18:51-61

28. Doran SJ, Charles-Edwards L, Reinsberg SA, et al. A complete distortion correction for MR images: I. Gradient warp correction. Phys Med Biol. 2005; 50:1343-61.

29. Zelefsky M, Leibel S, Gaudin P, et al. Dose escalation with three-dimensional conformal radiation therapy affects the outcome in prostate cancer. Int J Radiat Oncol Bio. Phys. 1998:41:491-500. 